\title{
PREVENÇÃO DE QUEDAS EM IDOSOS A PARTIR DO AUTOCUIDADO: UMA REVISÃO BIBLIOGRÁFICA
}

\author{
PREVENTION OF FALLS IN ELDERLY FROM THE SELF-CARE: A LITERATURE \\ REVIEW
}

Anamaria Donato de Castro Petito

Faculdade de Enfermagem, FACER Faculdades Unidade de Ceres-GOanamariapetito@gmail.com

\section{Leticia Fernanda Xavier Costa}

Faculdade de Enfermagem, FACER Faculdades Unidade de Ceres-GO leticiafernandafotos@hotmail.com

\section{Guilherme Petito}

Faculdade de Enfermagem, FACER Faculdades Unidade de Ceres-GO guilherme.petito@hotmail.com

\section{Fabia Aparecida Alves de Souza}

Faculdade de Enfermagem, FACER Faculdades Unidade de Ceres-GO fabiacrv@gmail.com

Endereço para correspondência: Av. Brasil, s/n, qd. 13 Morada verde ceres - Go, Fone: (62) 3323-1040, E-mail: anamariapetito@gmail.com

\section{RESUMO:}

Introdução: A luta para manter o bem-estar e enfrentar o adoecimento é incessante no cotidiano daqueles que envelhecem. Os idosos são naturalmente mais vulneráveis aos múltiplos redutores da saúde, entre eles, a queda. Objetivo: Levantar produções científicas sobre a importância do autocuidado visando a prevenção de quedas em idosos. Metodologia: A pesquisa foi realizada através de revisão bibliográfica, com busca sistemática, buscando artigos publicados na Biblioteca Virtual de Saúde (BVS), tendo como base de dados a Literatura Latino-Americana e do Caribe em Ciências da Saúde (LILACS) e a Base de Dados de Enfermagem (BDENF) publicados entre 2005 e 2015, os quais debatiam sobre a "Prevenção de quedas em idosos a partir do autocuidado". Resultados e Discussão: Foram selecionados no total 16 artigos que compõem este estudo. $\mathrm{O}$ autocuidado fundamentado na Teoria de Orem se mostrou uma ferramenta essencial para o gerenciamento de quedas. Levando em consideração que aumento da idade, uma auto percepção negativa da saúde, o sedentarismo e fatores ambientais são as principais causas de quedas em idosos, a atuação do enfermeiro através do autocuidado é essencial para diminuir esses índices. Considerações finais: Foi constatado que o autocuidado fundamentado em uma análise crítica das necessidades e particularidades de cada cliente é instrumento essencial para redução do índice de quedas em idosos.

Descritores: Enfermagem; Idoso; Queda; Autocuidado e Teoria de Orem.

\section{ABSTRACT}

Introduction: The struggle to maintain the welfare and face the illness is relentless in everyday life of the aging population. The elderly are naturally more vulnerable to multiple reducing health, among them the fall. Objective: Raise scientific papers on the 
importance of self-care aimed at preventing falls in the elderly. Methodology: The survey was conducted through literature review, with systematic search, looking for articles published in the Virtual Health Library (VHL), whose database the Latin American and Caribbean Health Sciences (LILACS) and the Base Nursing Database (BDENF) published between 2005 and 2015, which debated on the "Prevention of falls in the elderly from the self-care." Results and Discussion: There were selected in total 16 articles that make up this study. The self-care based on Orem's theory proved an essential tool for managing outages. Considering that increasing age a self negative perception of health, physical inactivity and environmental factors are the main causes of falls in the elderly, the work of nurses through self-care is essential to reduce these rates. Final Thoughts: It was found that the self-care based on a critical analysis of the needs and particularities of each customer is essential tool for reducing the rate of falls in the elderly.

Descriptors: nursing; elderly; fall; Self-care and Orem's Theory.

\section{INTRODUÇÃO}

A luta para manter o bem-estar e enfrentar o adoecimento é incessante no cotidiano daqueles que envelhecem, sendo fundamental favorecer a saúde e incentivar a independência desses indivíduos. Para que isso ocorra é preciso saber lidar com as mudanças decorrentes do envelhecer (SOUZA, 2007).

A compreensão do que se constitui o bom envelhecer contribui para uma reflexão a respeito de um cuidar e agir que corresponda às necessidades e interesses das pessoas idosas (SILVA; SANTOS, 2010). O autocuidado idealizado por Dorothea Elizabeth Orem, se baseia em uma prática de atividades desenvolvidas pelo próprio indivíduo em seu benefício, seja para satisfazer suas necessidades fisiológicas ou comportamentais (SANTOS et al., 2012).

As alterações que ocorrem com o aumento da idade, podem afetar diretamente a vida e a saúde dos idosos, implicando em sua capacidade física e mental de exercer determinadas atividades do dia-a-dia (OLIVEIRA; ROZENDO, 2014). O enfermeiro, sendo entendedor dessas alterações, precisa estar atento para identificá-las. Ele deve saber compreender as necessidades evidentes ou não de cada cliente, realizando cuidados individualizados e buscando conservar a sua liberdade e autonomia (VALCARENGHI et al., 2014).

O processo de envelhecimento é influenciado por várias circunstâncias, como doenças, particularidades biológicas e causas externas. Entre estas a queda se destaca por se tratar de um evento erroneamente considerado normal e próprio do processo de 
envelhecimento. Quedas podem ser na verdade um indicador da perda total do equilíbrio postural estando relacionada à insuficiência súbita dos mecanismos neurais e osteoarticulares envolvidos na manutenção da postura (FABRICIO; RODRIGUES; JUNIOR, 2004).

No Brasil, cerca de $30 \%$ dos idosos já sofreram pelo menos uma queda no último ano, sendo a maioria acima de 85 anos (PIMENTEL; SHEICHER, 2009; SIQUEIRA et al., 2007). Quedas nesse estágio da vida podem ser originadas por fatores intrínsecos como alterações fisiológicas do envelhecimento, doenças e uso de fármacos, e fatores extrínsecos decorrentes de circunstâncias sociais e ambientais. O reconhecimento dos fatores associados às quedas auxiliam no esclarecimento de fenômenos causais, consequentemente em medidas profiláticas (GAI et al., 2010, RIBEIRO et al., 2008).

O Diagnóstico de Enfermagem (DE) para risco de quedas, baseado no North American Nursing Diagnosis Association (NANDA), é definido como a suscetibilidade aumentada de quedas que podem causar prejuízo físico e fragilidade do paciente e tem sido usado como base de planejamento na prevenção destes casos. A Nursing Interventions Classification (NIC) institui, inclusive, precauções especiais para pacientes com esse diagnóstico (LUZIA; ALMEIDA; LUCENA, 2014).

Um novo olhar para o cuidar, leva os trabalhadores da saúde a assistirem o idoso de forma geral e mais alerta nos acontecimentos do processo de envelhecimento através de sua avaliação multidimensional (TIER; LUNARDI; SANTOS, 2008). Nesse processo é importante que o enfermeiro desenvolva uma parceria com a família, incentivando o autocuidado e adequando os seus conhecimentos à realidade do assistido (PEREIRA, 2012). Um dos grandes desafios que norteiam as ações do setor de saúde atualmente é justamente este: o de promover, de maneira competente e adequada, assistência às necessidades de saúde do idoso e a capacitação de recursos humanos especializados para essa área (CASTRO; FIGUEIREDO, 2009).

Desta forma fica indispensável as ações do enfermeiro e da equipe de enfermagem para realizar cuidados voltados a prevenção de quedas em pessoas idosas, estejam elas hospitalizadas, institucionalizadas ou em suas casas (FREITAS et al., 2011).

Portanto, este trabalho teve como principal objetivo levantar produções científicas sobre a importância do autocuidado visando a prevenção de quedas nos idosos. Assim 
como, destacar a necessidade do autocuidado de acordo com a Teoria de Orem, identificando em publicações as principais causas de quedas em idosos levando em consideração a atuação do enfermeiro para prevenção de quedas a partir do autocuidado.

\section{METODOLOGIA}

Tratou-se de um estudo descritivo qualitativo, realizado através de uma revisão bibliográfica com busca sistemática, que se propôs a investigar a "Prevenção de quedas em idosos a partir do autocuidado". Os artigos utilizados na pesquisa foram retirados da Biblioteca Virtual de Saúde (www.bvsalud.com), tendo como base de dados a Literatura Latino-americana e do Caribe em Ciências da Saúde (LILACS) e a Base de Dados de Enfermagem (BDENF).

A coleta de dados foi realizada empregando-se os seguintes descritores de forma combinada: Enfermagem; idoso; queda; autocuidado e Teoria de Orem. Foram admitidos apenas artigos na língua portuguesa, relacionados ao assunto proposto e publicados entre 2005 à 2015, foram excluídos as publicações que não foram revisadas por pares (Teses, dissertações, folhetos, etc.) e a duplicidade de informação, conforme o fluxograma (Figura 1):

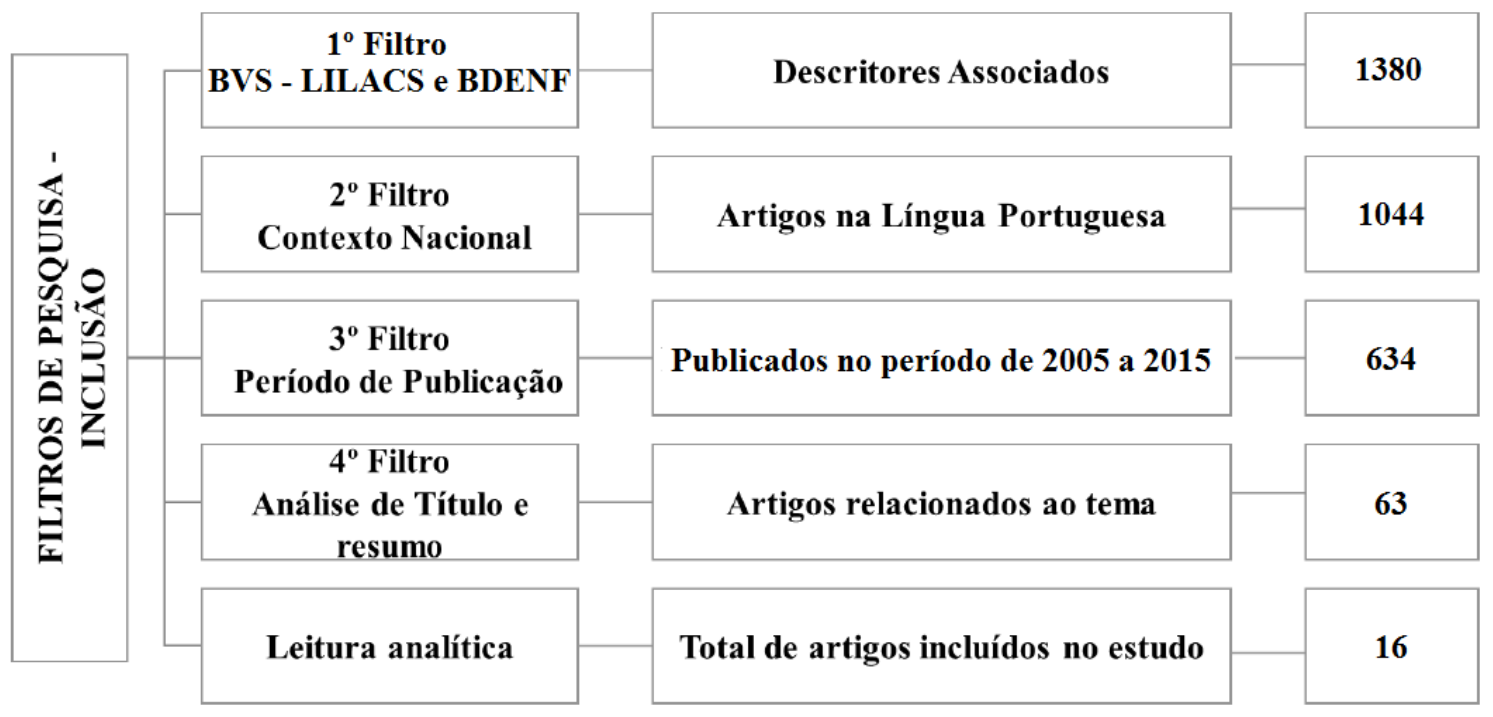

Figura 1: Trajetória metodológica dos artigos pesquisados 


\section{RESULTADOS E DISCUSSÃO}

A tabela 1 é composta por 5 estudos que expõem a necessidade do autocuidado a partir da Teoria de Orem. Matéria de estudo dos enfermeiros, desenvolvida em 1959 por Dorothea Elizabeth Orem. Segundo tal princípio o autocuidado se caracteriza pela capacidade de uma pessoa praticar seu próprio cuidado, em todas as áreas de forma independente. A partir desse fundamento pode ser determinado com maior clareza quando a enfermagem se faz necessária e quando o paciente pode desenvolver seu próprio cuidado (COSTA; CASTRO; ACIOLI, 2013).

Na perspectiva de Orem, é essencial que haja uma análise da condição do adulto que resulta na necessidade de ações por parte do profissional de enfermagem, seja pela ausência da sua habilidade, para manter um autocuidado contínuo quando na recuperação de doença ou lesão ou, de maneira geral, para sustentação da sua vida e saúde. Dessa forma, com implantação de uma prática de cuidar estruturada e teoricamente fundamentada, é possível traçar um plano que atenda de forma individualizada e coerente as necessidades de cada cliente (RAIMONDO et al., 2012).

As dificuldades enfrentadas no seguimento da terapêutica apontam o impacto dos sintomas da depressão, a vulnerabilidade e a falta de conhecimento sobre o tratamento como prejudiciais a sua competência para o autocuidado. A Teoria de Orem preconiza a utilização do sistema de apoio e educação como instrumento do cuidar, tendo o enfermeiro responsabilidade tanto no informar quanto na recuperação, na prevenção e no reintegrar do cliente na sociedade (SANTOS; SARAT, 2008; IBANEZ et al., 2014).

Orem afirma ainda que o envolvimento familiar é substancial para garantir a qualidade de vida do assistido. Estudos defendem a importância da presença e do cuidado da família no tratamento do doente. A Teoria estabelece que o profissional da saúde, principalmente o enfermeiro tem papel significante no conciliar dessas duas partes para que nem o cuidado nem o cuidador fiquem em segundo plano (SANTOS; SARAT, 2008; COSTA; CASTRO, 2014).

O autocuidado deve ser empregado em grupos vulneráveis, por estimular a melhora da qualidade de vida através das atividades diárias. Um dos fatores que classificam a fragilidade do idoso é sarcopenia e a astenia por aumentarem a possibilidade de queda (COSTA; CASTRO; ACIOLI, 2013; COSTA; CASTRO, 2014) 
Após a busca sistematizada, identifica-se estudos relacionados às principais causas de quedas em idosos na tabela 2. As quedas tem aumentado significativamente nos últimos anos devido ao crescimento dessa população. Por se tratar de casos geralmente mais graves, que necessitam com mais frequência de internação hospitalar e consumirem mais recursos do que os de qualquer outro grupo etário, a traumatologia geriátrica tem assumindo um papel cada vez mais importante para o setor da saúde. Nota-se que, entre os vários fatores que contribuem para ocorrência de quedas, o aumento da idade e uma autopercepção negativa da saúde são os que mais elevam a sua frequência, (BIAZIN; RODRIGUES, 2009; ALMEIDA et al., 2012).

Quedas são definidas como episódios de desequilíbrio que levam o indivíduo ao chão. O equilíbrio é produto da harmonia entre os sistemas vestibular, visual, somatossensorial e musculoesquelético e, com o envelhecimento, esses sistemas tendem a sofrer perdas funcionais que retardam a resposta motora responsável pela manutenção da postura e do equilíbrio corporal. Neste contexto analisar as causas mais recorrentes de quedas em idosos é primordial para impedir o aumento dos níveis de morbidade e mortalidade, como consequência de fraturas (ALMEIDA et al., 2012).

No que se refere ao gênero, foi observado que mulheres sofrem maior número de quedas, não estando esclarecidos, contudo, os mecanismos que explicam esse processo. Especula-se que por apresentarem quantidade de massa magra e força muscular menor do que a dos homens da mesma idade, somado a uma perda mais acentuada de massa óssea em consequência da redução de estrógeno, aumentando a probabilidade de osteoporose e uma maior exposição a atividades domésticas, as idosas são mais susceptíveis a esse risco (CRUZ et al., 2012). 
1 Tabela 1: Necessidade do autocuidado de acordo com a Teoria de Orem

\begin{tabular}{|c|c|c|c|c|c|}
\hline AUTOR & TÍTULO DO ARTIGO & OBJETIVO & CONCLUSÃO & ANO & PERIÓDICO \\
\hline $\begin{array}{l}\text { SANTOS; } \\
\text { SARAT }\end{array}$ & $\begin{array}{l}\text { Modalidades de aplicação da } \\
\text { teoria do autocuidado de Orem } \\
\text { em comunicações científicas } \\
\text { de enfermagem brasileira }\end{array}$ & $\begin{array}{l}\text { Analisar publicações de enfermagem } \\
\text { aplicando a Teoria do Autocuidado de } \\
\text { Orem. }\end{array}$ & $\begin{array}{l}\text { A Teoria de Orem foi aplicada como } \\
\text { referencialna identificação do déficit de } \\
\text { autocuidado como instrumento do cuidar. }\end{array}$ & 2008 & $\begin{array}{l}\text { Rev. enferm. } \\
\text { UERJ }\end{array}$ \\
\hline $\begin{array}{l}\text { RAIMONDO et } \\
\text { al }\end{array}$ & $\begin{array}{l}\text { Produção científica brasileira } \\
\text { fundamentada na Teoria de } \\
\text { Enfermagem de Orem: revisão } \\
\text { integrativa }\end{array}$ & $\begin{array}{l}\text { Sumarizar a produção científica brasileira } \\
\text { fundamentada na Teoria de Enfermagem de } \\
\text { Orem }\end{array}$ & $\begin{array}{l}\text { A teoria de Orem vem sendo utilizada } \\
\text { como base teórico-filosófica para } \\
\text { fundamentar e salientar o papel do } \\
\text { enfermeiro no cuidado. }\end{array}$ & 2012. & Rev. bras. Enferm. \\
\hline $\begin{array}{l}\text { COSTA; } \\
\text { CASTRO; } \\
\text { ACIOLI }\end{array}$ & $\begin{array}{l}\text { Capacidade de autocuidado de } \\
\text { adultos e idosos } \\
\text { hospitalizados: implicações } \\
\text { para o cuidado de enfermagem }\end{array}$ & $\begin{array}{l}\text { Classificar e avaliar esse grupo quanto ao } \\
\text { grau de dependência em relação ao } \\
\text { autocuidado,conforme a concepção teórica } \\
\text { de Dorothea Orem }\end{array}$ & $\begin{array}{l}\text { O aumento do número de pacientes com } \\
\text { processos crônicos que envolvem graus } \\
\text { de dependência demanda o } \\
\text { desenvolvimento de ações sistematizadas } \\
\text { de enfermagem com adultos e idosos que } \\
\text { evidenciam a síndrome do déficit de } \\
\text { autocuidado e graus de dependência }\end{array}$ & 2013 & $\begin{array}{l}\text { REME - Revista } \\
\text { Mineira de } \\
\text { Enfermagem. }\end{array}$ \\
\hline $\begin{array}{l}\text { COSTA; } \\
\text { CASTRO }\end{array}$ & $\begin{array}{l}\text { Autocuidado do cuidador } \\
\text { familiar de adultos ou idosos } \\
\text { dependentes após a alta } \\
\text { hospitalar }\end{array}$ & $\begin{array}{l}\text { Apresentar uma compreensão sobre o } \\
\text { autocuidado do cuidador familiar segundo a } \\
\text { teoria de Dorothea Orem }\end{array}$ & $\begin{array}{l}\text { O apoio de familiares contribuiu como } \\
\text { facilitador das estratégias para o } \\
\text { autocuidado do cuidador. }\end{array}$ & 2014 & Rev. bras. enferm \\
\hline IBANEZ et al & $\begin{array}{l}\text { Adesão e dificuldades } \\
\text { relacionadas ao tratamento } \\
\text { medicamentoso em pacientes } \\
\text { com depressão }\end{array}$ & $\begin{array}{l}\text { Verificar a adesão e conhecimento de } \\
\text { pessoas com depressão quanto à } \\
\text { farmacoterapia prescrita, a satisfação com a } \\
\text { equipe de saúdee as dificuldades } \\
\text { relacionadas ao tratamento medicamentoso. }\end{array}$ & $\begin{array}{l}\text { Foram apontados o impacto dos sintomas } \\
\text { depressivos sobre o autocuidado, a } \\
\text { insatisfação com efeitos dos } \\
\text { psicofármacos, a carência de apoio, a } \\
\text { polifarmacoterapia e a falta de } \\
\text { conhecimento sobre o transtorno e } \\
\text { tratamento. }\end{array}$ & 2014 & Rev. bras. enferm. \\
\hline
\end{tabular}


Estudos revelam que a maior prevalência de quedas é causada por fatores extrínsecos decorrentes de circunstâncias sociais e ambientais e sedentarismo e os por fatores intrínsecos como alterações fisiológicas do envelhecimento, doenças e uso de fármacos ocorrem em menor proporção. Em estudo de comparação entre idosos institucionalizados ou não, os que se encontram em instituições apresentam, mais fatores de risco para quedas, como maior polimedicação, equilíbrio precário e queixas de tontura, provavelmente provenientes da falta de atividades físicas (BATISTA; OLIVEIRA; OLIVEIRA, 2009; BORGES; GARCIA; RIBEIRO, 2009; LOJUDICE et al., 2010).

Os autores divergem e retratando que as disfunções comuns ao avanço da idade prevenção desse fenômeno também pode contribuir para que ele aconteça (CRUZ et al., 2012).

Na tabela 3 encontra-se a atuação do enfermeiro para prevenção de quedas a partir do autocuidado, evidenciando a importância desse profissional no zelo e manutenção da saúde. A ele é atribuído a responsabilidade ética, legal e técnica do cuidar do ser humano, tanto no atendimento primário quanto nos secundário e terciário: na atenção básica de saúde, hospitalar ou domiciliar, abrangendo o atendimento do indivíduo, família e comunidade (MENEZES; PRIEL; PEREIRA, 2011).

A regulamentação do exercício de enfermagem está estabelecida desde 1986 pelo Conselho Federal de Enfermagem (COFEN) na lei $n^{\circ} 7.498$, onde a mesma coloca o planejamento da assistência como uma determinação do profissional de enfermagem. Desde 2002 a implantação da Sistematização da Assistência de Enfermagem (SAE) é obrigatória em todas as unidades de saúde, sendo essa uma ação exercida exclusivamente pelo enfermeiro em todos os seus componentes (BRASIL, 2002)

A efetivação da SAE vem sendo cada vez mais utilizada. Esta metodologia científica permite a implantação de uma prática assistencial adequada, uma organização de ideias de forma mais técnico-científica e humana e o direcionamento dos cuidados de enfermagem, obtendo assim melhor assistência pelos profissionais de enfermagem (TANURE; PINHEIRO, 2011). 
1 Tabela 2: Principais causas de quedas em idosos

\begin{tabular}{|c|c|c|c|c|c|}
\hline AUTOR & TÍTULO DO ARTIGO & OBJETIVO & CONCLUSÃO & ANO & PERIÓDICO \\
\hline $\begin{array}{l}\text { BATISTA; } \\
\text { OLIVEIRA; } \\
\text { OLIVEIRA }\end{array}$ & $\begin{array}{l}\text { Quedas e fatores de risco } \\
\text { em idosos } \\
\text { institucionalizados }\end{array}$ & $\begin{array}{l}\text { Identificar a prevalência de quedas e os fatores } \\
\text { de risco em idosos residentes em instituições } \\
\text { de longa permanência }\end{array}$ & $\begin{array}{l}\text { No estudo realizado houve uma prevalência de } \\
\text { quedas entre idosos com idade acima de } 75 \\
\text { anos, sendo a maioria destas causadas por } \\
\text { fatores extrínsecos e idosos sedentários. }\end{array}$ & 2009 & Terapia Manual \\
\hline $\begin{array}{l}\text { BIAZIN; } \\
\text { RODRIGUES, }\end{array}$ & $\begin{array}{l}\text { Perfil dos idosos que } \\
\text { sofreram trauma em } \\
\text { Londrina }\end{array}$ & $\begin{array}{l}\text { Caracterizar os idosos que sofreram trauma e } \\
\text { foram internados nos três hospitais terciários, } \\
\text { da cidade de Londrina - Paraná. }\end{array}$ & $\begin{array}{l}\text { A queda foi onsevada como a principal causa } \\
\text { de trauma, a qual pode ser evitada observando } \\
\text { os fatores intrínsecos e os extrínsecos que a } \\
\text { predispõem, com o intuito de estabelecer } \\
\text { estratégias de prevenção desse tipo de trauma. }\end{array}$ & 2009 & $\begin{array}{l}\text { Rev. Esc. } \\
\text { Enferm. USP }\end{array}$ \\
\hline $\begin{array}{l}\text { BORGES; } \\
\text { GARCIA; } \\
\text { RIBEIRO }\end{array}$ & $\begin{array}{l}\text { Características clínico- } \\
\text { demográficas, quedas e } \\
\text { equilíbrio funcional de } \\
\text { idosos institucionalizados } \\
\text { e comunitários }\end{array}$ & $\begin{array}{l}\text { Avaliar o equilíbrio funcional, os fatores de } \\
\text { risco e a prevalência de quedas entre idosos } \\
\text { institucionalizados e comunitários }\end{array}$ & $\begin{array}{l}\text { Apesar de o estudo reforçar a presença de } \\
\text { múltiplos fatores de risco para quedas, a maior } \\
\text { instabilidade postural nos idosos } \\
\text { institucionalizados destaca a importância } \\
\text { clínica de se considerar as diferenças entre } \\
\text { esses idosos e os comunitários. }\end{array}$ & 2009 & $\begin{array}{l}\text { Fisioterapia em } \\
\text { Movimento }\end{array}$ \\
\hline LOJUDICE et al & $\begin{array}{l}\text { Quedas de idosos } \\
\text { institucionalizados: } \\
\text { ocorrência e fatores } \\
\text { associados }\end{array}$ & $\begin{array}{l}\text { Verificar a ocorrência de quedas em idosos } \\
\text { institucionalizados e identificar seus fatores } \\
\text { associados. }\end{array}$ & $\begin{array}{l}\text { Os resultados apontam para a necessidade da } \\
\text { implementação de programas de prevenções de } \\
\text { quedas em instituições asilares. }\end{array}$ & 2010 & $\begin{array}{l}\text { Rev. Bras. } \\
\text { Geriatr. } \\
\text { Gerontol }\end{array}$ \\
\hline ALMEIDA et al. & $\begin{array}{l}\text { Análise de fatores } \\
\text { extrínsecos e intrínsecos } \\
\text { que predispõem a quedas } \\
\text { em idosos }\end{array}$ & $\begin{array}{l}\text { Analisar, em uma amostra de idosos de Porto } \\
\text { Alegre, RS, os fatores intrínsecos e extrínsecos } \\
\text { que predispõem ao risco de queda e fraturas. }\end{array}$ & $\begin{array}{l}\text { Foram observados que os fatores intrínsecos } \\
\text { que predispõem ao risco de quedas e fraturas } \\
\text { são: faixa etária mais elevada; autopercepção } \\
\text { ruim da visão e autopercepção ruim da saúde; } \\
\text { já os fatores extrínsecos são o tipo de moradia e } \\
\text { a renda mensal igual ou inferior a um salário- } \\
\text { mínimo. }\end{array}$ & 2012 & $\begin{array}{l}\text { Assoc. Med. } \\
\text { Bras }\end{array}$ \\
\hline CRUZ et al & $\begin{array}{l}\text { Prevalência de quedas e } \\
\text { fatores associados em } \\
\text { idosos }\end{array}$ & $\begin{array}{l}\text { Estimar a prevalência de quedas em idosos e } \\
\text { analisar fatores associados }\end{array}$ & $\begin{array}{l}\text { O conhecimento dos fatores associados à } \\
\text { ocorrência desse evento pode auxiliar na } \\
\text { elaboração de estratégias de prevenção e } \\
\text { serviços de saúde adequados. }\end{array}$ & 2012 & $\begin{array}{l}\text { Rev. Saúde } \\
\text { Pública }\end{array}$ \\
\hline
\end{tabular}


É fundamental oferecer a esse grupo um ambiente seguro para o exercício de suas

2 atividades cotidianas. É tarefa do enfermeiro e da equipe de enfermagem priorizar a acessibilidade dessas pessoas através do estudo de propostas de ação para prevenção de acidentes por quedas (FREITAS et al., 2011).

A implantação de protocolos focados no autocuidado para prevenção de quedas em hospitais, incluindo, entre outras intervenções, o treinamento direcionado dos funcionários envolvidos na assistência ao cliente, mostrou ser uma alternativa bastante eficiente para o problema. Essa mediação tem refletido de maneira positiva na qualidade de vida dos assistidos e tem se tornado ferramenta essencial para o enfermeiro no processo de melhoria da qualidade e da segurança no atendimento ao idoso (CORREA et al., 2012).

Estudos apontam que uma internação hospitalar de qualidade por si só já pode desempenhar um papel importante na prevenção de quedas. Uma vez que o autocuidado especializado e direcionado da equipe de enfermagem soma para o melhoramento do desempenho funcional do idoso, diminuindo a influência negativa dos fatores ambientais para o risco de queda. Os impactos da hospitalização na capacidade funcional dos grupos estudados mostraram-se positivos em sessenta por cento dos casos (IZZO; GRÜNER, 2005). partir de ações que proporcionem o autocuidado. Do mesmo modo, a criação de um modelo de atenção ao idoso que tenha como foco a identificação de potenciais riscos e direcionamento de recursos do sistema de saúde para uma intervenção precoce resulta em melhores chances de reabilitação e redução do impacto na sua funcionalidade (DIAS et al., 2015).

Ao analisar os estudos relacionados a enfermagem utilizando o autocuidado na prevenção de quedas, observou-se uma escassez de publicações. Sendo preocupante, pois a enfermagem baseada em evidências, necessita de bases para sua aplicabilidade. Estudos utilizando o processo de enfermagem na prevenção de quedas não foram encontrados, evidenciando a necessidade de trabalhos epidemiológicos para esclarecimento relacionados ao tema. 
1 Tabela 3:Atuação do enfermeiro para prevenção de quedas a partir do autocuidado

\begin{tabular}{|c|c|c|c|c|c|}
\hline AUTOR & TÍTULO DO ARTIGO & OBJETIVO & CONCLUSÃO & ANO & PERIÓDICO \\
\hline $\begin{array}{l}\text { IZZO; } \\
\text { GRÜNER }\end{array}$ & $\begin{array}{l}\text { Impacto da internação } \\
\text { hospitalar na capacidade } \\
\text { funcional do idoso em } \\
\text { enfermaria geriátrica }\end{array}$ & $\begin{array}{l}\text { Investigar o impacto da internação hospitalar na } \\
\text { independência funcional dos idosos e traçar o perfil } \\
\text { sócio demográfico e clínico dos mesmos }\end{array}$ & $\begin{array}{l}\text { O impacto da internação hospitalar } \\
\text { na capacidade funcional do grupo } \\
\text { mostrou-se positivo uma vez que } 60 \\
\text { por cento obtiveram melhora do seu } \\
\text { desempenho funcional. }\end{array}$ & 2005 & $\begin{array}{l}\text { Mundo saúde } \\
\text { (Impr.) }\end{array}$ \\
\hline $\begin{array}{l}\text { FREITAS et } \\
\quad \text { al }\end{array}$ & $\begin{array}{l}\text { Cuidado de enfermagem } \\
\text { para prevenção de quedas } \\
\text { em idosos: proposta para } \\
\text { ação }\end{array}$ & $\begin{array}{l}\text { Construir proposta de ação de enfermagem para } \\
\text { prevenção de quedas em idosos }\end{array}$ & $\begin{array}{l}\text { Elaborou-se proposta de ação de } \\
\text { enfermagem voltada à prevenção de } \\
\text { quedas, segundo as necessidades } \\
\text { emergentes: quedas em idosas e } \\
\text { alimentação saudável; quedas da } \\
\text { própria altura e ambiente seguro; } \\
\text { fratura e fortalecimento do sistema } \\
\text { musculoesquelético. }\end{array}$ & 2011. & $\begin{array}{l}\text { Rev. bras. } \\
\text { enferm. }\end{array}$ \\
\hline $\begin{array}{l}\text { MENEZES; } \\
\text { PRIEL; } \\
\text { PEREIRA }\end{array}$ & $\begin{array}{l}\text { Autonomia e } \\
\text { vulnerabilidade do } \\
\text { enfermeiro na prática da } \\
\text { Sistematização da } \\
\text { Assistência de } \\
\text { Enfermagem. }\end{array}$ & $\begin{array}{l}\text { Reconhecer a autonomia e a vulnerabilidade do } \\
\text { enfermeiro no processo de implantação e } \\
\text { implementação da Sistematização da Assistência de } \\
\text { Enfermagem (SAE) }\end{array}$ & $\begin{array}{l}\text { Destacou-se a autonomia do } \\
\text { enfermeiro no agir com liberdade e } \\
\text { responsabilidade, na tomada de } \\
\text { decisão com base científica e na } \\
\text { conquista do valor de seu trabalho } \\
\text { social. }\end{array}$ & 2011 & $\begin{array}{l}\text { Revista da } \\
\text { Escola de } \\
\text { Enfermagem da } \\
\text { USP.. }\end{array}$ \\
\hline $\begin{array}{l}\text { CORREA et } \\
\text { al }\end{array}$ & $\begin{array}{c}\text { Implantação de um } \\
\text { protocolo para } \\
\text { gerenciamento de quedas } \\
\text { em hospital: resultados de } \\
\text { quatro anos de seguimento }\end{array}$ & $\begin{array}{l}\text { Apresentar os resultados de um protocolo de } \\
\text { gerenciamento de quedas implantado em um hospital } \\
\text { privado na cidade de São Paulo, Brasil }\end{array}$ & $\begin{array}{l}\text { As ações realizadas refletiram no } \\
\text { índice de quedas e a caracterização } \\
\text { dos eventos permitiu redirecionar } \\
\text { intervenções voltadas aos pacientes } \\
\text { mais susceptíveis e ao reforço das } \\
\text { ações educacionais. }\end{array}$ & 2012 & $\begin{array}{l}\text { Revista da } \\
\text { Escola de } \\
\text { Enfermagem da } \\
\text { USP. }\end{array}$ \\
\hline DIAS et al & $\begin{array}{l}\text { Estratégias para humanizar } \\
\text { o cuidado com o idoso } \\
\text { hospitalizado: estudo com } \\
\text { enfermeiros assistenciais }\end{array}$ & $\begin{array}{l}\text { Investigar as estratégias que enfermeiros assistenciais } \\
\text { empregam para assistir o idoso hospitalizado }\end{array}$ & $\begin{array}{l}\text { Este estudo evidencia o } \\
\text { compromisso dos enfermeiros } \\
\text { participantes da pesquisa no que } \\
\text { tange ao cuidado humanizado de } \\
\text { enfermagem direcionado ao idoso } \\
\text { hospitalizado. }\end{array}$ & 2015 & $\begin{array}{l}\text { Rev. pesqui. } \\
\text { cuid. fundam. } \\
\text { (Online) }\end{array}$ \\
\hline
\end{tabular}




\section{CONSIDERAÇÕES FINAIS}

O estudo possibilitou reconhecer o autocuidado com base na Teoria de Orem como uma ferramenta essencial no gerenciamento de quedas, constatando que o bom cuidado se fundamenta na análise crítica das necessidades de cada cliente e que o seu tratamento deve ser pensado de forma a atender essas particularidades.

Percebe-se que quedas em idosos acontecem, sobretudo, devido ao aumento da idade e uma autopercepção ruim da saúde. Nota-se ainda que circunstâncias sociais, ambientais e o sedentarismo possuem influência significativa no aumento desse risco. Foi observado que entre os gêneros, as mulheres costumam sofrer maior número de quedas presumindo-se que isso ocorra por apresentarem quantidade mais baixa de massa magra e força muscular, perda mais acentuada de massa óssea e estarem mais expostas a atividades domésticas. Além disso o não recebimento de orientações para prevenção desse fenômeno também pode contribuir para que ele aconteça.

Foi demonstrada também a importância da atuação do enfermeiro, traçando planos que atendam de forma individualizada e coerente as necessidades de cada indivíduo, informando quanto a recuperação, na prevenção de quedas a partir do autocuidado e no reintegrar do cliente na sociedade. Porém não foi possível identificar como o processo de enfermagem influencia a prevenção de quedas à partir dos diagnósticos de enfermagem (autocuidado), pela escassez de publicações.

\section{REFERÊNCIAS BIBLIOGRÁFICAS}

ALMEIDA, S.T.; SOLDERA, C.L.C.; CARLI, G.A.; GOMES, I.; RESENDE, T.L. Análise de fatores extrínsecos e intrínsecos que predispõem a quedas em idosos. Rev. Assoc. Med. Bras., São Paulo, v. 58, n. 4, p. 0104-4230, jul.- ago. 2012.

BATISTA, P.K.O.; OLIVEIRA, D.A.A.P.; OLIVEIRA, R.F. Quedas e fatores de risco em idosos institucionalizados. Terapia Manual. v. 7, n. 34, p. 745-480, nov.-dez. 2009.

BIAZIN, T.D.; RODRIGUES, R.A.P. Perfil dos idosos que sofreram trauma em Londrina - Paraná. Rev. Esc. Enferm. USP. São Paulo., v. 43 n. 3, 2009.

BRASIL. CONSELHO FEDERAL DE ENFERMAGEM. Lei 7498/86, de 25 de junho de 1986. Dispõe sobre a regulamentação do exercício da enfermagem. Brasília: 1986. Disponível em <http://www.portalcofen.gov.br >. Acesso em18 maio 2013. 
CONSELHO FEDERAL DE ENFERMAGEM. Lei n²72/2002. Dispõe sobre a sistematização da assistência de enfermagem nas Instituições de Saúde Brasileiras. Brasília:2002. Disponível em < http://www.cofen.gov.br/resoluo-cofen-2722002revogada-pela-resoluao-cofen-n-3582009_4309.html>.

BORGES; L.L.; GARCIA, P.A.; RIBEIRO, S.O.V. Características clínico-demográficas, quedas e equilíbrio funcional de idosos institucionalizados e comunitários. Fisioterapia em Movimento. v. 22, n.1, p. 53-60, jan.-mar. 2009.

CASTRO, M.R; FIGUEIREDO, N.M.A. O estado da arte sobre cuidado do idoso. PhusisRevista de Saúde Coletiva. v.19, n.3, p. 743-759. Rio de Janeiro, 2009.

CORREA, A.D.; MARQUES, I.A.B.; MARTINEZ, M.C.; LAURINO, P.S.; LEÃO, E.R.; CHIMENTÃO, D.M.N. Implantação de um protocolo para gerenciamento de quedas em hospital: resultados de quatro anos de seguimento. Revista da Escola de Enfermagem da USP. Rev. esc. enferm. USP, São Paulo, v.46, n.1, 2012.

COSTA, S.R.D.; CASTRO, E.A.B. Autocuidado do cuidador familiar de adultos ou idosos dependentes após a alta hospitalar. Rev. bras. enferm. Brasília, v.67, n. 6, 2014.

COSTA, S.R.D.; CASTRO, E.A.B.; ACIOLI, S. Capacidade de autocuidado de adultos e idosos hospitalizados: implicações para o cuidado de enfermagem. REME - Revista Mineira de Enfermagem. v. 17, n. 1, p. 192-199, jan.-mar., 2013.

CRUZ, D.T.; RIBEIRO, L.C.; VIEIRA, M.T.; TEIXEIRA, M.T.B.; BASTOS, R.R.; LEITE, I.C.G. Prevalência de quedas e fatores associados em idosos. Rev. Saúde Pública. São Paulo., v. 46, n. 1, feb. 2012.

DIAS, K.C.C.O.; LOPES, M.E.L.; FRANÇA, I.S.X.; BATISTA, P.S.S.; BATISTA, J.B.V. SOUSA, F.S. Estratégias para humanizar o cuidado com o idoso hospitalizado: estudo com enfermeiros assistenciais. Rev. pesqui. cuid. fundam. (Online); v. 7, n. 1, p.:1832-1846, jan.-mar. 2015.

FABRICÍO, S.C.C.; RODRIGUES, R.A.P.; JUNIOR, M.L.C. Causas e consequências de quedas de idosos atendidos em hospital público. Rev. Saúde Pública. v.38, n.1, p. 93-99. São Paulo, 2004.

FREITAS, R.; SANTOS, S.S.C.; HAMMERSHMIDT, K.S.A.; SILV A, M.E.; PELZER, M.T. Cuidado de enfermagem para prevenção de quedas em idosos: proposta para ação. Rev. bras. enferm. Brasília. v.64, n.3, may.- jun., 2011. 
GAI, J; GOMES, L; NÓBREGA, O.T.N; RODRIGUES, M.P. Fatores associados a quedas em mulheres idosas residentes na comunidade. Revista AssocMed Bras. v.56, n.3, p.327-32. Brasília, 2010.

IBANEZ, G.; MERCEDES, B.P.C.; VEDANA, K.G.G.; MIASSO, A.I. Adesão e dificuldades relacionadas ao tratamento medicamentoso em pacientes com depressão. Rev. bras. enferm. Brasília, v.67, n.4, jul.-aug. 2014.

IZZO, H.; GRÜNER, T. Impacto da internação hospitalar na capacidade funcional do idoso em enfermaria geriátrica. Mundo saúde (Impr.); v. 29, n. 4, p. 629-638, out.-dez. 2005.

LOJUDICE, D.C.; LAPREGA, M.R.; RODRIGUES, R.A.P.; JÚNIOR, A.L.R. Quedas de idosos institucionalizados: ocorrência e fatores associados. Rev. Bras. Geriatr. Gerontol. Rio de Janeiro, v.13, n. 3, 2010.

LUZIA, M.F; ALMEIDA, M.A; LUCEMA, A.F. Mapeamento de cuidados de enfermagem para pacientes com risco de quedas na NurcingInterventionsClassification.RevistaEscEnferm USP. v.48, n.4, p.632-9. Rio Grande do Sul, 2014.

MENEZES, S.R.T.; PRIEL, M.R.; PEREIRA, L.L. Autonomia e vulnerabilidade do enfermeiro na prática da Sistematização da Assistência de Enfermagem. Revista da Escola de Enfermagem da USP. São Paulo, v.45, n.4, 2011.

OLIVEIRA, J.M.; ROZENDO, C.A. Instituição de longa permanência para idosos: um lugar de cuidado para quem não tem opção? Rev. Bras. Enferm. v.67, n.5, p. 773-779, 2014.

PEREIRA, M. R. A promoção do autocuidado na pessoa em processo de transição. 2012. 154 f. Dissertação (Mestrado em enfermagem da reabilitação ) Escola Superior de Enfermagem da Reabilitação, Coimbra.

PIMENTEL, R.M; SCHEICHER, M.E. Comparação do risco de queda em idosos sedentários e ativos por meio da escala de equilíbrio de Rerg. Fisioterapia e pesquisa. v.16, n.1, p. 6-10. São Paulo, 2009.

RAIMONDO, M.L.;FEGADOLI, D. MÉIER, M.J.; WALL, M.L.; LANRONICI, L.M.; FERRAZ, M.I.R. Produção científica brasileira fundamentada na Teoria de Enfermagem de Orem: revisão integrativa. Rev. bras. enferm. Brasília, v.65, n. 3, may.-june. 2012. 
RIBEIRO, A.P; SOUZA, E.R; ATIE, S; SOUZA, A.C; SCHILITHZ, A.O. A influência das quedas na qualidade de vida dos idosos. Ciência e Saúde coletiva. v.13, n.4, p. 1265 - 1273. Rio de Janeiro, 2008.

SANTOS, Z.M.S.A; MARTINS, J.O; FROTA, N.M; CAETANO, J.A; MOREIRA, R.A.N; BARROSA, L.M. Autocuidado universal praticado por idosos em uma instituição de longa permanência. Rev. Bras. Geriatr. v.15, n.4, p. 747-754. Rio de Janeiro, 2012.

SANTOS, I.; SARAT, C.N.F. Modalidades de aplicação da teoria do autocuidado de Orem em comunicações científicas de enfermagem brasileira.Rev. enferm. UERJ, Rio de Janeiro, v. 16, n. 3, p. 313-318, jul.- ago. 2008.

SILVA, A.C.S; SANTOS, I. Promoção do autocuidado de idosos para o envelhecer saudável: aplicação da teoria de Nola Pender. Texto \& Contexto - Enfermagem. Florianópolis, v. 19, n. 4, p. 745-753, 2010.

SIQUEIRA, F.V; FACCHINI, L.A; PICCINI, R; TOMASI, E; THUMÉ, E; SILVEIRA, D .S; VIEIRA, V; HALLAL, P.C. Prevalência de quedas em idosos e fatores associados. Rev. Saúde Pública. v.41, n.5, p.749-56. Rio Grande do Sul, 2007.

SOUZA, A.F.; MURAI, H.C. Qualidade de vida e envelhecimento. Revista de Enfermagem da UNISA. v.8, p. 9-11, 2007.

TANNURE, MC; PINHEIRO, AM. SAE: Sistematização da Assistência de Enfermagem. Guia Prático. 2nd ed. Rio de Janeiro: Guanabara Koogan: 2011

TIER, C.G; LUNARDI, V.L; SANTOS, S.S.C. Cuidado ao idoso à luz da complexidade. Revista Eletrônica de Enfermagem. v.10, n.2, p.530-536. 2008.

VALCARENGHI, R.V; SANTOS, S.S.C; HAMMERSCHMIDT, K.S.A.; BARLEM, E.L.D.; GOMES, G.C.; SILVA, B.T. Ações institucionais alicerçadas em diagnósticos de enfermagem para prevenção de quedas em idosos. Revista Rene. v.15, n.2, p. 224-232, 2014. 